PROCEEDINGS OF THE

AMERICAN MATHEMATICAL SOCIETY

Volume 126, Number 7, July 1998, Pages 2103-2115

S 0002-9939(98)04410-4

\title{
EVADING PREDICTORS WITH CREATURES
}

\author{
OTMAR SPINAS
}

(Communicated by Andreas R. Blass)

\begin{abstract}
We continue the theory of evasion and prediction which was introduced by Blass and developed by Brendle, Shelah, and Laflamme. We prove that for arbitrary sufficiently different $f, g \in \omega_{\omega}$, it is consistent to have $\mathfrak{e}_{g}<\mathfrak{e}_{f}$, where $\mathfrak{e}_{f}$ is the evasion number of the space $\prod_{n<\omega} f(n)$. For this we apply a variant of Shelah's "creature forcing".
\end{abstract}

\section{INTRODUCTION}

In [B], Blass introduced the concept of evasion and prediction in order to study a certain property of subgroups of $\left(\mathbf{Z}^{\omega},+\right)$, called the Specker phenomenon. A predictor for the space ${ }^{\omega} \omega$ is a family $\Pi=\left(\pi_{n}: n \in D\right)$ such that $D \in[\omega]^{\omega}$ and $\pi_{n}:{ }^{n} \omega \rightarrow \omega$. Then $\Pi$ is said to predict $x \in{ }^{\omega} \omega$ if for all except finitely many $n \in D$, $\pi_{n}(x\lceil n)=x(n)$; otherwise $x$ evades $\Pi$. The evasion number $\mathfrak{e}$ is defined as the minimal cardinality of a set $X \subseteq{ }^{\omega} \omega$ such that for every predictor $\Pi$, some $x \in X$ evades $\Pi$. It is easy to see that $\omega_{1} \leq \mathfrak{e} \leq \mathfrak{c}$ holds. Blass proved various relations between $\mathfrak{e}$ and the standard cardinal invariants of the continuum.

In [Br], Brendle continued this investigation, and he discovered that this concept is related to yet another algebraic phenomenon, namely the existence of Gross spaces, i.e. a certain kind of vector spaces which are equipped with a bilinear form, which had previously been studied extensively (see [Sp], [ShSp1]). Brendle also started to develop a more general theory of evasion and prediction. Given any $f \in{ }^{\omega} \omega$, we can analogously define a predictor for the space $\prod_{n<\omega} f(n)=\left\{x \in{ }^{\omega} \omega\right.$ : $(\forall n) x(n)<f(n)\}$ as a familiy $\left(\pi_{n}: n \in D\right)$ such that $\pi_{n}: \prod_{i<n} f(i) \rightarrow f(n)$. The corresponding evasion number $\mathfrak{e}_{f}$ is then defined as the minimal cardinality of a set $X \subseteq \prod_{n<\omega} f(n)$ with the property that every predictor for $\prod_{n<\omega} f(n)$ is evaded by some $x \in X$. By $\prod_{i<n} f(i)$ above we meant the cartesian product of natural numbers taken as sets of their predecessors. However, below we will sometimes use the same notation for the arithmetic product.

It is clear that for $f \leq g$ we have that $\mathfrak{e} \leq \mathfrak{e}_{g} \leq \mathfrak{e}_{f}$. Brendle (see [Br, Lemma 2, p.525]) proved that for constant $f, \mathfrak{e}_{f}$ is constant, and he denoted the common value by $\mathfrak{e}_{f i n}$. Moreover, he defined $\mathfrak{e}_{u b d}$, the unbounded evasion number, as $\min \left\{\mathfrak{e}_{f}: f \in\right.$ $\left.{ }^{\omega} \omega\right\}$. In [Br, p. 526], Brendle proved the consistency of $\mathfrak{e}<\mathfrak{e}_{u b d}$ and of $\mathfrak{e}_{u b d}<\mathfrak{e}_{\text {fin }}$.

Received by the editors November 27, 1995 and, in revised form, December 10, 1996.

1991 Mathematics Subject Classification. Primary 03E05, 03E35.

Partially supported by a research fellowship of the Alexander von Humboldt Foundation.

(c)1998 American Mathematical Society 
It is natural to continue this work and try to distinguish between $\mathfrak{e}_{f}$ and $\mathfrak{e}_{g}$ for $f, g$ which are not constant. Note that unless $\lim _{n \rightarrow \infty} f(n)=\infty$ we have $\mathfrak{e}_{f}=\mathfrak{e}_{\text {fin }}$. This follows from the following easy fact.

Fact. If $f, g \in{ }^{\omega} \omega$ and there exists some injective $\pi \in{ }^{\omega} \omega$ with $g=f \circ \pi$, then $\mathfrak{e}_{g} \leq \mathfrak{e}_{f}$.

It is also clear that for every $k<\omega$ we have $\mathfrak{e}_{f+k}=\mathfrak{e}_{f}$, where $f^{+k}(n)=f(k+n)$. In this paper we build models for $\mathfrak{e}_{g}<\mathfrak{e}_{f}$ for arbitrary $f, g$, supposing that $g$ grows sufficiently faster than $f$, that is, in our terminology defined in Definition 1.5 below, $g$ runs away from $P(f)$, where $P(f)$ is a certain function definable from and larger than $f$. For this we apply a certain kind of forcing which was invented by Shelah and which was used by him and his coauthors to produce a number of very delicate consistency proofs (see [Sh1], [Sh2], [GSh], [RSh1], [RSh2]). Conditions in these forcings are trees carrying logarithmic measures, objects which Shelah likes to call "creatures". We use a standard countable support iteration of length $\omega_{2}$ and therefore get $\mathfrak{c}=\omega_{2}$, and so only two possible values for $\mathfrak{e}_{g}$ and $\mathfrak{e}_{f}$.

In [GSh], certain cardinal invariants $\mathfrak{c}(f, g)$ are investigated which are in a sense dual to the cardinals $\mathfrak{b}(f, g)$ defined in Definition 1.2 below. By using a countable support product the authors can make the continuum arbitrarily large and produce $\aleph_{1}$ different cardinals of the form $\mathfrak{c}(f, g)$ (by an unpublished result of Shelah, even continuum many). The question arises whether a similar approach can be taken to produce many different $\mathfrak{b}(f, g)$ 's and, since these are related to evasion numbers (see Theorem 1.4 below), many different evasion numbers $\mathfrak{e}_{f}$. Although there exist uncountable families $\left(f_{\alpha}: \alpha<\kappa\right)$ such that either $f_{\alpha}$ runs away from $P\left(f_{\beta}\right)$ or conversely, whenever $\alpha \neq \beta$, it seems to be a difficult problem. The main difference from [GSh] is that the logarithmic measures used here are more difficult to handle than those in [GSh], which are essentially counting measures. What seems to be necessary for applying a product construction instead of an iteration are certain Ramsey properties of the measures, which are shared by counting measures but not ours.

\section{Slaloms PRODUCing PREDiCtors}

Definition 1.1. Given $f \in \omega^{\omega} \omega$ with $f(n)>0$ for all $n<\omega$, an $f$-slalom is a function $S: \omega \rightarrow[\omega]^{<\omega}$ such that $|S(n)|=f(n)$ for all $n<\omega$.

Definition 1.2. Let $f, g \in{ }^{\omega} \omega$ with $0<f(n)<g(n)$ for all $n<\omega$. Define cardinal invariants as follows:

$$
\begin{gathered}
\mathfrak{b}(f, g)=\min \left\{|X|: X \subseteq \prod_{n<\omega} g(n) \wedge(\forall S)(S \text { is an } f \text {-slalom }\right. \\
\left.\left.\quad \Rightarrow(\exists x \in X)\left(\exists^{\infty} n\right) x(n) \notin S(n)\right)\right\}, \\
\begin{aligned}
\mathfrak{b}_{0}(f, g)=\min \left\{|X|: X \subseteq \prod_{n<\omega} g(n) \wedge(\forall S)(S \text { is an } f \text {-slalom }\right. \\
\left.\left.\Rightarrow\left(\forall A \in[\omega]^{\omega}\right)(\exists x \in X)\left(\exists^{\infty} n \in A\right) x(n) \notin S(n)\right)\right\} .
\end{aligned}
\end{gathered}
$$

It is easy to see that $\omega_{1} \leq \mathfrak{b}(f, g) \leq \mathfrak{b}_{0}(f, g) \leq \mathfrak{c}$ holds.

Definition 1.3. Let $I_{n}=\left[\frac{n(n+1)}{2}, \frac{n(n+1)}{2}+n\right]$. For $f \in{ }^{\omega} \omega$ define $P(f) \in{ }^{\omega} \omega$ by $P(f)(n)=\prod_{i \in I_{n}} f(i)$. Let $\bar{d} \in{ }^{\omega} \omega$ be the diagonal, i.e. $\bar{d}(n)=n$. 
Note that $\left(I_{n}: n<\omega\right)$ is a partition of $\omega$ into adjacent intervals such that the $n$th interval $I_{n}$ has size $n+1$. Note that if $f(n) \geq 2$ always, then $P(f)>n$ for all $n<\omega$. The following theorem is essentially proved in [B].

Theorem 1.4. $\mathfrak{b}_{0}(\bar{d}, P(f)) \leq \mathfrak{e}_{f}$, for all $f \in \omega_{\omega}^{\omega}$ with $f(n) \geq 2$ for all $n<\omega$.

Proof. Let $X \subseteq \prod_{n<\omega} f(n)$ with $|X|<\mathfrak{b}_{0}(\bar{d}, P(f))$. We have to find a predictor which predicts $X$. Let $I_{n}$ be as in Definition 1.3. For $x \in X$ let $\bar{x}=\left(x \uparrow I_{n}: n<\omega\right)$. Then $\bar{x} \in \prod_{n<\omega} P(f)(n)$. Since $|\{\bar{x}: x \in X\}|<\mathfrak{b}_{0}(\bar{d}, P(f))$, there exist a $\bar{d}$-slalom $S$ and $A \in[\omega]^{\omega}$ such that for all $x \in X$, for almost all $n \in A, \bar{x}(n) \in S(n)$. We may interpret $S(n)$ as a subset of the $(n+1)$-dimensional $\mathbf{Q}$-vector space $\mathbf{Q}^{I_{n}}$. Since $|S(n)|=n$, there exists a non-zero linear functional $c^{n}: \mathbf{Q}^{I_{n}} \rightarrow \mathbf{Q}$ which annihilates every vector in $S(n)$. For $i \in I_{n}$ let $e_{i} \in \mathbf{Q}^{I_{n}}$ be the unit vector defined by $e_{i}(j)=\delta_{i j}$ (Kronecker), let $c_{i}^{n}=c^{n}\left(e_{i}\right)$, and let $d_{n}=\max \left\{i \in I_{n}: c_{i}^{n} \neq 0\right\}$. It is then clear that for every $x \in X$ and $n \in A$ with $\bar{x}(n) \in S(n)$, rewriting $c^{n}(\bar{x}(n))=0$, we have

$$
x\left(d_{n}\right)=-\frac{1}{c_{d_{n}}^{n}} \sum_{i \in I_{n} \cap d_{n}} c_{i}^{n} \cdot x(i) .
$$

Therefore, if we define a predictor $\Pi=\left(\pi_{d}: d \in D\right)$ by $D=\left\{d_{n}: n<\omega\right\}$ and

$$
\pi_{d_{n}}(\sigma)=-\frac{1}{c_{d_{n}}^{n}} \sum_{i \in I_{n} \cap d_{n}} c_{i}^{n} \cdot \sigma(i)
$$

then $\Pi$ predicts $X$.

Definition 1.5. For functions $f, g \in{ }^{\omega} \omega$ with $f(n) \geq 2$ for all $n<\omega$, we say that $g$ runs away from $f$ if there exists $A \in[\omega]^{\omega}$ with increasing enumeration $\left(a_{n}: n<\omega\right)$ such that the following holds: If we let

$$
\begin{gathered}
N_{1}(n)=\left[\prod_{0<i \leq n}\left(\begin{array}{c}
f\left(a_{i}\right) \\
a_{i}
\end{array}\right)\right]^{n^{2}}, \\
N_{2}(n)=1+g\left(a_{n}\right)^{\prod_{i<a_{n}} g(i)}, \\
N_{3}(n)=2^{\prod_{0<i \leq n}\left(\begin{array}{c}
f\left(a_{i}\right) \\
a_{i}
\end{array}\right)},
\end{gathered}
$$

and $C(n)=N_{2}(n)^{n \cdot N_{1}(n)} \cdot N_{3}(n)^{n}$, then for all $d, n<\omega$ the following hold:

(i) $\log _{C(n)} a_{n+1}>n$;

(ii) if $a_{n}<d \leq a_{n+1}$, then $\prod_{i<d} g(i) \cdot \prod_{i \leq n+1}\left(\begin{array}{c}f\left(a_{i}\right) \\ a_{i}\end{array}\right)^{a_{n}}<g(d)$.

Remark 1.6. For every $f \in \omega_{\omega}$ with $f(n) \geq 2$ for all $n<\omega$ there exists $g$ which runs away from $f$. (See the introduction for a stronger result.)

Definition 1.7. Assuming that $f, g, A$ and $C(n)$ are as in Definition 1.5, we define a logarithmic measure \|\|$_{n+1}$ on $\left[f\left(a_{n+1}\right)\right]^{a_{n+1}}$ as follows: For $X \subseteq\left[f\left(a_{n+1}\right)\right]^{a_{n+1}}$ let

$$
\|X\|_{n+1}=\max \left\{k \in \omega:\left(\forall s \subseteq f\left(a_{n+1}\right)\right)\left(|s|=C(n)^{k} \Rightarrow(\exists t \in X) s \subseteq t\right)\right\} .
$$

Remark 1.8. (1) Note that \|\|$_{n+1}$ is $C(n)$-complete, i.e., if $X=\bigcup_{i<C(n)} X_{i}$, there exists $i<C(n)$ such that $\left\|X_{i}\right\|_{n+1} \geq\|X\|_{n+1}-1$.

(2) By 1.5(i) we conclude that if $f(n) \geq n$ always, then $\left\|\left[f\left(a_{n+1}\right)\right]^{a_{n+1}}\right\|_{n+1} \geq n$ for all $n<\omega$. 
Our main result is the following theorem:

Theorem 1.9. Let $f, g \in{ }^{\omega} \omega$ be such that $f(n) \geq 2$ for all $n<\omega$ and $g$ runs away from $P(f)$. Then there exists a model of ZFC where $\mathfrak{e}_{g}<\mathfrak{e}_{f}$.

Remark 1.10. Note that by the remarks before and after the Fact from the Introduction, by Theorem 1.9, for every $f \in{ }^{\omega} \omega$ we can get $g \in{ }^{\omega} \omega$ such that consistently $\mathfrak{e}_{g}<\mathfrak{e}_{f}$.

The proof of Theorem 1.9 will be given in the following two sections. We will construct a forcing $Q(P(f), g, A)$, where $A$ witnesses that $g$ runs away from $P(f)$, such that if we force over a model $V$ for $C H$ with a countable support iteration of $Q(P(f), g, A)$ of length $\omega_{2}$, then in the extension $\mathfrak{b}_{0}(\bar{d}, P(f))=\omega_{2}$ is true, but on the other hand there is no predictor for the space $\prod_{n<\omega} g(n)$ which predicts $\prod_{n<\omega} g(n) \cap V$, and therefore $\mathfrak{e}_{g}=\omega_{1}$. By Theorem 1.4, this suffices. The forcing $Q(P(f), g, A)$ will add a generic $\bar{d}$-slalom $S$ with domain $A$ such that for every $x \in \prod_{n<\omega} P(f)(n) \cap V$, for almost all $n \in A, x(n) \in S(n)$.

\section{Creating CReatures}

Definition 2.1. Given $f, g \in{ }^{\omega} \omega$ such that $f \geq \bar{d}$ and $g$ runs away from $f$, as witnessed by $A=\left\{a_{0}<a_{1}<a_{2}<\ldots\right\}$, define a forcing $Q(f, g, A)$ as follows: Conditions are subtrees $p$ of the tree

$$
T=\left(\bigcup_{n<\omega} \prod_{0<i<n}\left[f\left(a_{i}\right)\right]^{a_{i}}, \subseteq\right),
$$

such that

(1) There exists $\sigma \in p$ such that for every $\tau \in p$ with $\sigma \subseteq \tau$, $\operatorname{succ}_{p}(\tau)$ has at least two elements, but $\operatorname{succ}_{p}\left(\sigma\lceil n)\right.$ has one element for all $n<|\sigma|$. Here, by $\operatorname{succ}_{p}(\sigma)$ we denote the set of all $x$ such that $\sigma^{\wedge}\langle x\rangle \in p$.

(2) For every branch $x$ of $p$ we have that $\lim _{n \rightarrow \infty}\left\|\operatorname{succ}_{p}(x \uparrow n)\right\|_{n+1}=\infty$.

The order on $Q(f, g, A)$ is inclusion.

Note that $\sigma$ as in (1) is unique. It will be denoted by $\operatorname{stem}(p)$. The collection of $\tau \in p$ which extends $\operatorname{stem}(\sigma)$ will be denoted by $p^{-}$. As usual, the set of branches of $p$ is denoted by $[p]$. The set of nodes of $p$ of length $j$ will be denoted by $p\lceil j$. Given $\sigma \in p$, let $p(\sigma)$ be the set of $\tau \in p$ which either extend $\sigma$ or are contained in $\sigma$.

Remark 2.2. It is easy to see that a filter which is $Q(f, g, A)$-generic over $V$ determines a slalom $S: A \rightarrow[\omega]^{<\omega}$ with $\left|S\left(a_{n}\right)\right|=a_{n}$ for all $a_{n} \in A$ such that for every $x \in \prod_{n<\omega} f(n) \cap V$, for almost all $a_{n} \in A, x\left(a_{n}\right) \in S\left(a_{n}\right)$. Moreover, from Lemma 2.4 below it easily follows that $Q(f, g, A)$ is proper. By standard results from [Sh3] it follows that a countable support iteration of $Q(f, g, A)$ of length $\omega_{2}$ has the $\aleph_{2^{-}}$ c.c. Therefore, if we iterate forcing with $Q(f, g, A) \omega_{2}$ times, $\mathfrak{b}_{0}(\bar{d}, f)=\omega_{2}$ will hold, and hence, if $f=P(h)$ for some $h$ with $h(n) \geq 2$ always, then by Theorem $1.4, \mathfrak{e}_{h}=\omega_{2}$ will hold. Hence we are left with proving that this iteration does not add a predictor for the space $\prod_{n<\omega} g(n)$ which predicts all $x \in \prod_{n<\omega} g(n) \cap V$. This will be proved in Corollary 3.7 below.

Definition 2.3. Let $p, q \in Q(f, g, A)$ and $j, k, N<\omega$ with $j \leq k$. Define:

(1) $q \leq^{*} p$ if $q \leq p$ and in addition, for all $\sigma \in q^{-}$,

$$
\left\|\operatorname{succ}_{q}(\sigma)\right\|_{|\sigma|+1} \geq\left\|\operatorname{succ}_{p}(\sigma)\right\|_{|\sigma|+1}-1
$$


(2) $q \leq^{*[j, k]} p$ if $q \leq^{*} p$ and for all $\sigma \in q^{-}$with $|\sigma| \notin[j, k]$ we have

$$
\operatorname{succ}_{q}(\sigma)=\operatorname{succ}_{p}(\sigma)
$$

(3) $q \leq^{*[j]} p$ if $q \leq{ }^{*[j, j]} p$;

(4) $q \leq_{N}^{*} p$ if $q \leq p$, for all $\sigma \in p$ with $\left\|\operatorname{succ}_{p}(\sigma)\right\|_{|\sigma|+1} \geq N$ we have

$$
\left\|\operatorname{succ}_{q}(\sigma)\right\|_{|\sigma|+1} \geq N
$$

and for all $\sigma \in p$ with $\left\|\operatorname{succ}_{p}(\sigma)\right\|_{|\sigma|+1}<N$ we have

$$
\operatorname{succ}_{p}(\sigma)=\operatorname{succ}_{q}(\sigma) .
$$

For the rest of this paper we fix $f, g \in{ }^{\omega} \omega$ such that $g$ runs away from $f$, as witnessed by $A \in[\omega]^{\omega}$, and we denote $Q(f, g, A)$ by $Q$.

Lemma 2.4. Suppose $p \in Q$ and $\tau$ is a $Q$-name for an element of $V$ and $N<\omega$. Then the following hold:

(1) There exist $q \leq_{N}^{*} p$ and $j<\omega$ such that for every $\sigma \in q\lceil j, q(\sigma)$ decides $\tau$.

(2) If there are no more than $C(n)$ possibilities for $\tau$, for some $n<\omega$, and if $q, j$ are as in (1), then for every $n \leq i \leq j$ there exists $q_{1} \leq^{*[i, j]} q$ such that for every $\sigma \in q_{1}\left\lceil i, q_{1}(\sigma)\right.$ decides $\tau$.

Proof. (1) is proved in the proof of [Sh2, Lemma 2.21]. (2) follows from the proof of [Sh2, Lemma 3.7]; in fact, the argument (decide a finite disjunction by shrinking levels of a condition in a downwards process, dropping norms by at most 1 , as long as the completeness of the logarithmic measures permits it) is one of the main features of "creature forcing", and it will used and explained in detail at many places throughout this paper.

Lemma 2.5. Let $\dot{d}, \pi_{\dot{d}}$ be $Q$-names such that $\Vdash_{Q}$ “d $\in \omega \wedge \pi_{\dot{d}}: \prod_{i<\dot{d}} g(i) \rightarrow g(\dot{d})$ ". Let $p \in Q$ and $j<\omega$ be such that for every $\sigma \in p \uparrow j, p(\sigma)$ decides $\dot{d}$ and $\pi_{\dot{d}}$, say as $d(\sigma)$ and $\pi(\sigma)$. Let $n^{*}$ be minimal such that $a_{n^{*}} \geq \min \{d(\sigma): \sigma \in p\lceil j\}$. Then there exists $q \leq^{*\left[n^{*}, j-1\right]} p$ such that for each $\sigma \in q\lceil j$ it is true that if we let $i^{*}=\min \left\{j, \min \left\{i: a_{i} \geq d(\sigma)\right\}\right\}$, then $q\left(\sigma\left\lceil i^{*}\right)\right.$ decides $\dot{d}$ and $\pi_{\dot{d}}$ (and so clearly as $d(\sigma)$ and $\pi(\sigma))$.

Proof. Fix $\nu \in p\left\lceil j-1\right.$. For each $d \leq a_{j-1}$ and $\pi: \prod_{i<d} g(i) \rightarrow g(d)$ let $A(d, \pi, \nu)=\left\{\sigma \in \operatorname{succ}_{p}(\nu): d(\sigma)=d \wedge \pi(\sigma)=\pi\right\}$, and let $B(\nu)=\left\{\sigma \in \operatorname{succ}_{p}(\nu)\right.$ : $\left.d(\sigma)>a_{j-1}\right\}$. Since \|\|$_{j}$ is $\left(\sum_{d \leq a_{j-1}}\left(\prod_{i<d} g(i)\right)^{g(d)}+1\right)$-complete, we may shrink $\operatorname{succ}_{p}(\nu)$ to either $B(\nu)$ or $A(d, \pi, \nu)$ for some $(d, \pi)$, dropping the norm by at most 1. We do this for all $\nu \in p\left\lceil j-1\right.$ and thus obtain a tree $p_{j} \leq^{*[j-1]} p$. We repeat this process at level $j-2$ of $p_{j}$, as follows. Let $\nu \in p_{j}\lceil j-2$. Note that for each $\sigma \in \operatorname{succ}_{p_{j}}(\nu)$, either $p_{j}(\sigma) \Vdash$ " $\dot{d}>a_{j-1}$ ", or else $p_{j}(\sigma)$ decides $\dot{d}$ and $\pi$, say as $d(\sigma), \pi(\sigma)$. For each $d \leq a_{j-2}$ and $\pi: \prod_{i<d} g(i) \rightarrow g(d)$ let $A(d, \pi, \nu) \subseteq \operatorname{succ}_{p_{j}}(\nu)$ be as defined above, and let $B(\nu)=\left\{\sigma \in \operatorname{succ}_{p_{j}}(\nu): p_{j}(\sigma) \Vdash " \dot{d}>a_{j-2} "\right\}$. As before we may shrink $\operatorname{succ}_{p_{j}}(\nu)$ for each $\nu \in p_{j}\left\lceil j-2\right.$ to obtain $p_{j-1} \leq^{*[j-2]} p_{j}$ with the property that for every $\nu \in p_{j-1}\left\lceil j-2\right.$, $\operatorname{succ}_{p_{j-1}}(\nu)$ equals either $B(\nu)$ or $A(d, \pi, \nu)$ for some $d, \pi$, and therefore we have that either $p_{j-1}(\nu) \Vdash " \dot{d}>a_{j-2}$ " or else $p_{j-1}(\nu)$ decides $\dot{d}$ and $\pi_{\dot{d}}$. In this fashion we continue until we obtain $p_{n^{*}+1} \leq^{*\left[n^{*}\right]} p_{n^{*}+2}$ by shrinking level $n^{*}$ of $p_{n^{*}+2}$ such that for all $\sigma \in p_{n^{*}+1}\left\lceil n^{*}\right.$, either $p_{n^{*}+1}(\sigma) \Vdash$ "d $>a_{n^{*}}$ ", or else $p_{n^{*}+1}$ decides $\dot{d}$ and $\pi_{\dot{d}}$. 
Now let $q=p_{n^{*}+1}$. It follows immediately from the construction that $q \leq *\left[n^{*}, j-1\right]$ $p$. Now let $\sigma \in q\left\lceil j\right.$ and let $i^{*}$ be defined as in the Lemma. By construction we have that for every $i^{*} \leq i \leq j$, either $q(\sigma \uparrow i)$ decides $\dot{d}$ and $\pi$, or else $q(\sigma \uparrow i) \Vdash " a_{i}<\dot{d}$ ". But the second alternative is impossible for $i^{*}$.

Corollary 2.6. Let $\dot{d}, \pi_{\dot{d}}, p, j, q, d(\sigma)$ and $\pi(\sigma)$ be as in Lemma 2.5. Then for each $d \in\{d(\sigma): \sigma \in q \uparrow j\}$ there is $k(d) \in g(d)$ such that for all $\sigma \in q \uparrow j$, if $d(\sigma)=d$, then $k(d) \notin \operatorname{ran}(\pi(\sigma))$. Therefore there exists $x \in \prod_{n<\omega} g(n)$ in $V$ such that $q \Vdash " \pi_{\dot{d}}(x \uparrow \dot{d}) \neq x(\dot{d}) "$.

Proof. Let $d=d(\sigma)$ for some $\sigma \in q \uparrow j$, and let $i^{*}=\min \left\{j, \min \left\{i: a_{i} \geq d\right\}\right\}$. By Lemma 2.5 , there are at most $\prod_{0<i \leq i^{*}}\left(\begin{array}{c}f\left(a_{i}\right) \\ a_{i}\end{array}\right)$ many functions $\pi: \prod_{i<d} g(i) \rightarrow g(d)$ such that $\pi=\pi(\sigma)$ for some $\sigma \in q \uparrow j$. Since by Definition 1.5(ii) we have that $\prod_{i<d} g(i) \cdot \prod_{0<i \leq i^{*}}\left(\begin{array}{c}f\left(a_{i}\right) \\ a_{i}\end{array}\right)<g(d)$, we can choose $k(d) \in g(d)$ not in the range of any $\pi(\sigma)$ with $\sigma \in q\lceil j$ and $d(\sigma)=d$. It is now easy to find $x$ as desired.

\section{ITERATING CREATURES}

Let $\left(P_{\alpha}, \dot{Q}_{\beta}: \alpha \leq \omega_{2}, \beta<\omega_{2}\right)$ be a countable support iteration of $Q=Q(f, g, A)$.

Definition 3.1. Let $p, q \in P_{\omega_{2}}, F \in[\operatorname{dom}(p)]^{<\omega}, j, k, N<\omega$. Let $\sigma_{\alpha} \in T$, for $\alpha \in F$. Define:

(1) $p$ is $(F, j)$-determined if for every $\alpha \in F, p\lceil\alpha$ decides $p(\alpha) \uparrow j$.

(2) By induction on $\alpha^{*}=\max (F)$ we define simultaneously when $\left(\sigma_{\alpha}: \alpha \in F\right)$ is $p$-possible of length $j$, and $p\left(\sigma_{\alpha}: \alpha \in F\right):\left(\sigma_{\alpha}: \alpha \in F\right)$ is p-possible of length $j$ if $\left(\sigma_{\alpha}: \alpha \in F \cap \alpha^{*}\right)$ is $p$-possible of length $j$, and $p\left(\sigma_{\alpha}: \alpha \in F \cap \alpha^{*}\right) \Vdash$ " $\sigma_{\alpha^{*}} \in$ $p\left(\alpha^{*}\right)\lceil j "$. In this case let

$$
p\left(\sigma_{\alpha}: \alpha \in F\right)=p\left(\sigma_{\alpha}: \alpha \in F \cap \alpha^{*}\right)\left\lceil\alpha ^ { * \wedge } p ( \alpha ^ { * } ) ( \sigma _ { \alpha ^ { * } } ) ^ { \wedge } p \left\lceil\left[\alpha^{*}+1, \omega_{2}\right) .\right.\right.
$$

(3) $q \leq_{F}^{*} p$ if for every $\alpha \in F, q\left\lceil\alpha \Vdash " q(\alpha) \leq^{*} p(\alpha)\right.$ ", and $p(\alpha)=q(\alpha)$ for $\alpha \notin F$.

(4) $q \leq_{F}^{*[j, k]} p$ if for every $\alpha \in F, q \uparrow \alpha \Vdash " q(\alpha) \leq{ }^{*[j, k]} p(\alpha) "$, and $p(\alpha)=q(\alpha)$ for $\alpha \notin F$.

(5) $q \leq_{F, N}^{*} p$ if $q \leq p$ and, for every $\alpha \in F, q\left\lceil\alpha \Vdash " q(\alpha) \leq_{N}^{*} p(\alpha) "\right.$.

Lemma 3.2. Let $p \in P_{\omega_{2}}, j, k<\omega$, and $F \in[\operatorname{dom}(p)]^{k}$ be such that $p$ is $(F, j)$ determined. Let $\tau$ be a $P_{\omega_{2}}$-name and $X \subseteq V$ be finite such that $p \Vdash$ " $\tau \in X$ ", and the size of $X$ is restricted as follows:

$$
|X|^{\left[\prod_{0<i \leq j}\left(\begin{array}{c}
f\left(a_{i}\right) \\
a_{i}
\end{array}\right)\right]^{k-1}} \leq C(j) .
$$

Moreover, suppose that for some $N<\omega$, for all $\alpha \in F$ we have that

$$
p\left\lceil\alpha \Vdash “ ( \forall i \geq j ) \left(\forall \sigma \in p(\alpha)\lceil i)\left\|\operatorname{succ}_{p(\alpha)}(\sigma)\right\|_{i+1} \geq N+2 " .\right.\right.
$$

Then there exists an $(F, j)$-determined $q \leq_{F, N}^{*} p$ such that for every $q$-possible $\left(\sigma_{\alpha}: \alpha \in F\right)$ of length $j, q\left(\sigma_{\alpha}: \alpha \in F\right)$ decides $\tau$, and moreover $q \uparrow \alpha \Vdash$ " $q(\alpha) \uparrow$ $j=p(\alpha) \uparrow j "$, for all $\alpha \in F$.

Proof. We prove it by induction on $\max (F)$. Let $\alpha=\max (F)$. Working in $V^{P_{\alpha}}$, there exists a $Q_{\alpha}$-name $q_{1}$ for a condition in $P_{\alpha+1, \omega_{2}}$ such that $\Vdash_{Q_{\alpha}} \quad$ " $q_{1} \leq p \uparrow$ $\left[\alpha+1, \omega_{2}\right) \wedge q_{1}$ decides $\tau "$. By Lemma 2.4 there exists $q(\alpha) \leq_{N}^{*} p(\alpha)$ with $q(\alpha) \uparrow$ $j=p(\alpha) \uparrow j$, such that for every $\sigma \in q(\alpha) \uparrow j, q(\alpha)(\sigma)$ decides how $q_{1}$ decides $\tau$. So in $V$ we have a $P_{\alpha}$-name for a function from $\prod_{0<i \leq j}\left(\begin{array}{c}f\left(a_{i}\right) \\ a_{i}\end{array}\right)$ into $X$ whose restriction 
to $q(\alpha)\left\lceil j\right.$ describes these decisions. There are no more than $|X|^{\prod_{0<i \leq j}\left(\begin{array}{c}f\left(a_{i}\right) \\ a_{i}\end{array}\right)}$ such functions, and

$$
\left[|X|^{\prod_{0<i \leq j}\left(\begin{array}{c}
f\left(a_{i}\right) \\
a_{i}
\end{array}\right)}\right]^{\left[\prod_{0<i \leq j}\left(\begin{array}{c}
f\left(a_{i}\right) \\
a_{i}
\end{array}\right)\right]^{k-2}}=|X|^{\left[\prod_{0<i \leq j}\left(\begin{array}{c}
f\left(a_{i}\right) \\
a_{i}
\end{array}\right)\right]^{k-1}} \leq C(j) .
$$

Therefore, by induction there exists some $(F \cap \alpha, j)$-determined $q_{0} \leq_{F \cap \alpha, N}^{*} p\lceil\alpha$ such that for every $\beta \in F \cap \alpha, q_{0}\left\lceil\beta \Vdash\right.$ " $q_{0}(\beta) \uparrow j=p(\beta)\lceil j$ ", and moreover for every $q_{0}$-possible $\left(\sigma_{\beta}: \beta \in F \cap \alpha\right)$ of length $j$ and every $\sigma \in q(\alpha)\lceil j$ as determined by $q_{0}\left(\sigma_{\beta}: \beta \in F \cap \alpha\right), q_{0}\left(\sigma_{\beta}: \beta \in F \cap \alpha\right)$ decides how $q(\alpha)(\sigma)^{\wedge} q_{1}$ decides $\tau$. Now let $q=q_{0} \wedge\langle q(\alpha)\rangle \wedge q_{1}$. Then $q$ is as desired.

Lemma 3.3. Let $p \in P_{\omega_{2}}, j_{0}, N<\omega, F \in[\operatorname{dom}(p)]<\omega$, and let $\tau$ be a $P_{\omega_{2}}$-name for an element of $V$. Then there exist $j_{1} \geq j_{0}$ and an $\left(F, j_{1}\right)$-determined $q \leq_{F, N}^{*} p$ such that for every q-possible $\left(\sigma_{\alpha}: \alpha \in F\right)$ of length $j_{1}, q\left(\sigma_{\alpha}: \alpha \in F\right)$ decides $\tau$, and, moreover, for all $\alpha \in F$ we have that

$$
q\left\lceil\alpha \Vdash " ( \forall i \geq j _ { 1 } ) \left(\forall \sigma \in q(\alpha)\lceil i)\left\|\operatorname{succ}_{q(\alpha)}(\sigma)\right\|_{i+1} \geq N " .\right.\right.
$$

Proof. By induction on $\max (F)$. Let $\alpha=\max (F)$. Work in $V^{P_{\alpha}}$ first. There we have a $Q_{\alpha}$-name $q_{1}$ for a condition in $P_{\alpha+1, \omega_{2}}$ below $p\left\lceil\left[\alpha+1, \omega_{2}\right)\right.$ such that $\Vdash_{Q_{\alpha}}$ " $q_{1}$ decides $\tau$ ". By Lemma 2.4, there exist $q(\alpha) \leq_{N}^{*} p(\alpha)$ and $j_{2} \geq j_{0}$ such that for every $\sigma \in q(\alpha) \uparrow j_{2}, q(\alpha)(\sigma)$ decides how $q_{1}$ decides $\tau$, and moreover for all $i \geq j_{2}$ and $\sigma \in q(\alpha)\left\lceil i,\left\|\operatorname{succ}_{q(\alpha)}(\sigma)\right\|_{i+1} \geq N\right.$. By the induction hypothesis there exist $j_{3} \geq j_{0}$ and $\left(F \cap \alpha, j_{3}\right)$-determined $q_{2} \leq_{F \cap \alpha, N}^{*} p\lceil\alpha$ such that for every $q_{2}$-possible $\left(\sigma_{\beta}: \beta \in F \cap \alpha\right)$ of length $j_{3}$ the following hold:

(1) $q_{2}\left(\sigma_{\beta}: \beta \in F \cap \alpha\right)$ decides $j_{2}$, say as $j_{2}\left(\sigma_{\beta}: \beta \in F \cap \alpha\right)$,

(2) $q_{2}\left(\sigma_{\beta}: \beta \in F \cap \alpha\right)$ decides $q(\alpha)\left\lceil j_{2}\right.$,

(3) $q_{2}\left(\sigma_{\beta}: \beta \in F \cap \alpha\right)$ decides the decision made about $\tau$ by $q(\alpha)(\sigma)^{\wedge} q_{1}$, for any $\sigma \in q(\alpha)\left\lceil j_{2}\right.$.

Let $j_{4}$ be the maximum over all the $j_{2}\left(\sigma_{\beta}: \beta \in F \cap \alpha\right)$. Now we may apply (part of) the induction hypothesis once more to obtain $j_{1} \geq \max \left\{j_{3}, j_{4},|F|-2\right\}$ and $\left(F \cap \alpha, j_{1}\right)$-determined $q_{3} \leq_{F \cap \alpha, N}^{*} q_{2}$ such that for all $\beta \in F \cap \alpha$ we have that

$$
q_{3}\left\lceil\beta \Vdash " ( \forall i \geq j _ { 1 } ) \left(\forall \sigma \in q_{3}(\beta)\lceil i)\left\|\operatorname{succ}_{q_{3}(\beta)}(\sigma)\right\|_{i+1} \geq N+2 " .\right.\right.
$$

Finally we apply Lemma 3.2 with $p=q_{3}, F=F \cap \alpha, j=j_{1}$, and $\tau=q(\alpha)\left\lceil j_{1}\right.$, to obtain $\left(F \cap \alpha, j_{1}\right)$-determined $q_{0} \leq_{F \cap \alpha, N}^{*} q_{3}$. For this, note that there are less than $N_{3}\left(j_{1}\right)$ possibilities for $\tau$, and by Definition 1.5 and the choice of $j_{1}$ we have

$$
N_{3}\left(j_{1}\right)^{\left[\prod_{0<i \leq j_{1}}\left(\begin{array}{c}
f\left(a_{i}\right) \\
a_{i}
\end{array}\right)\right]^{|F|-2}} \leq N_{3}\left(j_{1}\right)^{\left[\prod_{0<i \leq j_{1}}\left(\begin{array}{c}
f\left(a_{i}\right) \\
a_{i}
\end{array}\right)\right]^{j_{1}}} \leq C\left(j_{1}\right) .
$$

Then $j_{1}$ and $q=q_{0}^{\wedge} q(\alpha)^{\wedge} q_{1}$ are as desired.

Lemma 3.4. Suppose $p \in P_{\omega_{2}}, i^{*}<j<\omega, F \in[\operatorname{dom}(p)]^{<\omega}, p$ is $(F, j)$-determined and $|F|=i^{*}$. Then there exists $q \leq_{F \backslash\{\max (F)\}}^{*\left[i^{*}, j-1\right]}$ p such that $q$ is $(F, i)$-determined for every $i \in\left[i^{*}, j\right]$.

Proof. Let $F=\left\{\alpha_{0}<\alpha_{1}<\cdots<\alpha_{i^{*}-1}\right\}$. Fix some $p$-possible $\left(\sigma_{\alpha_{i}}: i<i^{*}-1\right)$ of length $j$, and let $\nu=\sigma_{\alpha_{i^{*}-2}}\lceil j-1$.

By $N_{3}(j-1)$-completeness of \|\|$_{j}$ we may shrink $\operatorname{succ}_{p\left(\alpha_{i^{*}-2}\right)}(\nu)$, dropping the norm by at most 1 , to some set $S$ such that $p\left(\alpha_{i^{*}-1}\right) \uparrow j$, as determined by $p\left(\sigma_{\alpha_{0}}, \ldots, \sigma_{\alpha_{i^{*}-3}}, \sigma\right) \uparrow \alpha_{i^{*}-1}$, is constant as we vary $\sigma \in S$. We do this shrinking 
for each $\operatorname{succ}_{p\left(\alpha_{i^{*}-2}\right)}(\nu)$, where for some $p$-possible $\left(\sigma_{\alpha_{0}}, \ldots, \sigma_{\alpha_{i^{*}-2}}\right)$ of length $j$, $\nu=\sigma_{\alpha_{i^{*}-2}}\left\lceil j-1\right.$, thus obtaining some $p_{i^{*}-2} \leq_{\left\{\alpha_{i^{*}-2}\right\}}^{*[j-1]} p$.

Now fix some $p_{i^{*}-2}$-possible $\left(\sigma_{\alpha_{i}}: i<i^{*}-2\right)$ of length $j$, and let $\nu=\sigma_{\alpha_{i^{*}-3}} \uparrow$ $j-1$. We want to shrink $\operatorname{succ}_{p\left(\alpha_{i^{*}-3}\right)}(\nu)$, dropping the norm by at most 1 , to some set $S$ such that

(1) $p\left(\alpha_{i^{*}-2}\right)\left\lceil j-1\right.$, as determined by $p\left(\sigma_{\alpha_{0}}, \ldots, \sigma_{\alpha_{i^{*}-4}}, \sigma\right)\left\lceil\alpha_{i^{*}-2}\right.$, is constant as we vary $\sigma \in S$, and

(2) $p\left(\alpha_{i^{*}-1}\right)\left\lceil j-1\right.$, as determined by $p\left(\sigma_{\alpha_{0}}, \ldots, \sigma_{\alpha_{i^{*}-4}}, \sigma, \rho\right)$, is constant as we vary $\sigma \in S$, for every $\rho \in p\left(\alpha_{i^{*}-2}\right)\lceil j-1$.

For this we need $N_{3}(j-1)\left(\prod_{0<i \leq j_{1}}\left(\begin{array}{c}f\left(a_{i}\right) \\ a_{i}\end{array}\right) N_{3}(j-1)\right)$-completeness of \|\|$_{j}$, which we have by Remark 1.8. By doing this shrinking for all $\operatorname{succ}_{p\left(\alpha_{i^{*}-3}\right)}(\nu)$ as above we obtain $p_{i^{*}-3} \leq_{\left\{\alpha_{i^{*}-3}^{*}[j-1]\right.} p_{i^{*}-2}$.

Continuing similarly, finally we obtain $p_{0} \leq_{\left\{\alpha_{0}\right\}}^{*[j-1]} p_{1}$. For this last step we will need that \|\|$_{j}$ is

$$
N_{3}(j-1)\left(\prod_{0<i \leq j_{1}}\left(\begin{array}{c}
f\left(a_{i}\right) \\
a_{i}
\end{array}\right) N_{3}(j-1)\right) \ldots\left(\left[\prod_{0<i \leq j_{1}}\left(\begin{array}{c}
f\left(a_{i}\right) \\
a_{i}
\end{array}\right)\right]^{i^{*}-2} N_{3}(j-1)\right) \text {-complete. }
$$

Hence we need

$$
N_{3}(j-1)^{i^{*}-1}\left(\prod_{0<i \leq j_{1}}\left(\begin{array}{c}
f\left(a_{i}\right) \\
a_{i}
\end{array}\right)\right)^{\frac{\left(i^{*}-2\right)\left(i^{*}-1\right)}{2}} \text {-completeness. }
$$

But this holds by Remark 1.8.

Let $p^{j-1}=p_{0}$. Then $p^{j-1}$ is $(F, i)$-determined for $i \in\{j, j-1\}$, and we have $p^{j-1} \leq_{F \backslash\{\max (F)\}}^{*[j-1]} p$. Continuing similarly, we construct

$$
p^{j-1} \geq_{\left\{\alpha_{i^{*}-2}\right\}}^{*[j-2]} p_{i^{*}-2}^{j-1} \ldots p_{1}^{j-1} \geq_{\left\{\alpha_{0}\right\}}^{*[j-2]} p_{0}^{j-1}
$$

and let $p^{j-2}=p_{0}^{j-1}$. Then $p^{j-2}$ is $(F, i)$-determined for $i \in\{j, j-1, j-2\}$, and

$$
p^{j-2} \leq_{F \backslash\{\max (F)\}}^{*[j-2]} p^{j-1} .
$$

Continuing similarly, we obtain a descending chain of conditions

$$
p \geq_{F \backslash\{\max (F)\}}^{*[j-1]} p^{j-1} \geq_{F \backslash\{\max (F)\}}^{*[j-2]} p^{j-2} \ldots p^{i^{*}+1} \geq_{F \backslash\{\max (F)\}}^{*\left[i^{*}\right]} p^{i^{*}} .
$$

Note that in the process of constructing $p^{i^{*}}$ from $p^{i^{*}+1}$ we need that \|\|$_{i^{*}+1}$ is

$$
N_{3}\left(i^{*}\right)^{i^{*}}\left(\prod_{0<i \leq i^{*}}\left(\begin{array}{c}
f\left(a_{i}\right) \\
a_{i}
\end{array}\right)\right)^{\frac{i^{*}\left(i^{*}+1\right)}{2}} \text {-complete. }
$$

This is true by remark 1.8. We let $q=p^{i^{*}}$. Then $q$ is as desired. We displayed the completeness of \|\|$_{i^{*}+1}$ needed to get $p^{i^{*}}$ rather than the general case (completeness of \|\|$_{i+1}$ needed to get $p^{i}$ ), since it is here that we need $|F| \leq i^{*}$, whereas in the general case we only need $|F| \leq i$.

Corollary 3.5. Let $\Pi=\left(\pi_{d}: d \in \dot{D}\right)$ be a $P_{\omega_{2}}$-name for a predictor for the space $\prod_{n<\omega} g(n)$. Let $(\dot{d}(n): n<\omega)$ be the increasing enumeration of $\dot{D}$, and let $p \in P_{\omega_{2}}$. There exist $q \leq p$ and sequences $\left(F_{n}: n<\omega\right)$ and $\left(j_{n}: n<\omega\right)$ such that the following hold:

(1) $\left|F_{n}\right|=n, F_{n} \subseteq F_{n+1}$ and $\bigcup_{n<\omega} F_{n}=\operatorname{dom}(q)$;

(2) $q$ is $\left(F_{n+1}, i\right)$-determined for every $i \in\left[a_{j_{n}}, j_{n+1}\right]$, for every $n$; 
(3) for every $q$-possible $\left(\sigma_{\alpha}: \alpha \in F_{n+1}\right)$ of length $j_{n+1}, q\left(\sigma_{\alpha}: \alpha \in F_{n+1}\right)$ decides $\dot{d}\left(a_{j_{n}}\right)$ and $\pi_{\dot{d}\left(a_{j_{n}}\right)}$, say as $d^{n}\left(\sigma_{\alpha}: \alpha \in F_{n+1}\right), \pi^{n}\left(\sigma_{\alpha}: \alpha \in F_{n+1}\right)$;

(4) $j_{n}<j_{n+1}$, and $j_{n+1}>\max \left\{d^{n}\left(\sigma_{\alpha}: \alpha \in F_{n+1}\right):\left(\sigma_{\alpha}: \alpha \in F_{n+1}\right)\right.$ is qpossible of length $\left.j_{n+1}\right\}$.

Proof. Using Lemma 3.3 and a standard bookkeeping, it is straightforward to construct sequences $\left(F_{n}: n<\omega\right),\left(j_{n}: n<\omega\right),\left(N_{n}: n<\omega\right)$ and $\left(q_{n}: n \in \omega\right)$ such that the following hold:

$(1)^{\prime}\left|F_{n}\right|=n, F_{n} \subseteq F_{n+1}$, and for every $\alpha \in \operatorname{dom}\left(q_{n}\right)$ there exists $m$ with $\alpha \in F_{m}$

$(2)^{\prime} q_{n}$ is $\left(F_{n}, j_{n}\right)$-determined and $q_{n+1} \leq_{F_{n+1}, N_{n+1}}^{*} q_{n}$;

$(3)^{\prime}$ for every $q_{n+1}$-possible $\left(\sigma_{\alpha}: \alpha \in F_{n+1}\right)$ of length $j_{n+1}, q_{n+1}\left(\sigma_{\alpha}: \alpha \in F_{n+1}\right)$ decides $\dot{d}\left(a_{j_{n}}\right)$ and $\pi_{\dot{d}\left(a_{j_{n}}\right)}$ as $d^{n}\left(\sigma_{\alpha}: \alpha \in F_{n+1}\right)$ and $\pi^{n}\left(\sigma_{\alpha}: \alpha \in F_{n+1}\right)$;

$(4)^{\prime} j_{n}<j_{n+1}$, and $j_{n+1}>\max \left\{d^{n}\left(\sigma_{\alpha}: \alpha \in F_{n+1}\right):\left(\sigma_{\alpha}: \alpha \in F\right)\right.$ is $q_{n+1^{-}}$ possible of length $\left.j_{n+1}\right\}$;

(5) $\left(\forall \alpha \in F_{n}\right) q\left\lceil\alpha \Vdash "\left(\forall i \geq j_{n}\right)\left(\forall \sigma \in q_{n}(\alpha)\lceil i)\left\|\operatorname{succ}_{q_{n}(\alpha)}(\sigma)\right\|_{i+1} \geq N_{n} "\right.\right.$;

(6) $N_{n+1}>N_{n}$ is large enough such that $q_{n+1} \leq_{F_{n+1}, N_{n+1}}^{*} q_{n}$ implies $q_{n+1} \uparrow \alpha$ $\Vdash$ " $q_{n+1}(\alpha) \uparrow j_{n}=q_{n}(\alpha) \uparrow j_{n} "$, for all $\alpha \in F_{n}$.

It is then easy to see that $\left(q_{n}: n<\omega\right)$ has an infimum, which we call $q_{0}$.

Next we construct $\left(q_{0}^{n}: n<\omega\right)$ such that $q_{0}^{0}=q_{0}, q_{0}^{n+1} \leq_{F_{n+1}}^{*\left[a_{j_{n}}, j_{n+1}-1\right]} q_{0}^{n}$, and $q_{0}^{n+1}$ is $\left(F_{n+1}, i\right)$-determined for every $i \in\left[a_{j_{n}}, j_{n+1}\right]$, for every $n<\omega$. This is possible by Lemma 3.4, using that $\left|F_{n+1}\right|=n+1<a_{j_{n}}$ for all $n$. Since by $(3)^{\prime}$ and $(4)^{\prime}$ we have that $j_{n+1} \geq a_{j_{n}}>j_{n}$ for all $n$, we conclude that $\left(q_{0}^{n}: n<\omega\right)$ has an infimum, which we call $q$. Then $q$ is as desired.

Lemma 3.6. Let $\dot{d}, \pi_{\dot{d}}$ be $P_{\omega_{2}}$-names such that $\Vdash_{P_{\omega_{2}}}$ "di $\in \omega \wedge \pi_{\dot{d}}: \prod_{i<\dot{d}} g(i) \rightarrow$ $g(\dot{d})$ ". Suppose $p \in P_{\omega_{2}}$ is $(F, j)$-determined and for every p-possible $\left(\sigma_{\alpha}: \alpha \in F\right)$ of length j, $p\left(\sigma_{\alpha}: \alpha \in F\right)$ decides $\dot{d}$ and $\pi_{\dot{d}}$, say as $d\left(\sigma_{\alpha}: \alpha \in F\right), \pi\left(\sigma_{\alpha}\right.$ : $\alpha \in F)$. Let $n^{*}$ be minimal such that $a_{n^{*}} \geq \min \left\{d\left(\sigma_{\alpha}: \alpha \in F\right):\left(\sigma_{\alpha}: \alpha \in\right.\right.$ $F)$ is p-possible of length $j\}$. Moreover assume that $p$ is even $(F, i)$-determined for all $i \in\left[n^{*}, j\right]$, and that $|F| \leq n^{*}$. Then there exists $q \leq_{F}^{*\left[n^{*}, j-1\right]} p$ such that for every q-possible $\left(\sigma_{\alpha}: \alpha \in F\right)$ of length $j$, if $i^{*}=\min \left\{j, \min \left\{i: a_{i} \geq d\left(\sigma_{\alpha}: \alpha \in F\right)\right\}\right\}$, then

$$
q\left(\sigma_{\alpha} \uparrow i^{*}: \alpha \in F\right) \Vdash “ \dot{d}=d\left(\sigma_{\alpha}: \alpha \in F\right) \wedge \pi_{\dot{d}}=\pi\left(\sigma_{\alpha}: \alpha \in F\right) " .
$$

Proof. Let $F=\left\{\alpha_{0}<\alpha_{1}<\cdots<\alpha_{n^{*}-1}\right\}$. The case $|F|<n^{*}$ is similar. By applying Lemma 2.5 to $p\left(\alpha_{n^{*}-1}\right)$ in $V^{P_{\alpha_{n}-1}}$ we obtain $q\left(\alpha_{n^{*}-1}\right) \leq{ }^{*\left[n^{*}, j-1\right]} p\left(\alpha_{n^{*}-1}\right)$ as there. Moreover, since $p$ is $(F, j)$-determined, for each $p$-possible $\left(\sigma_{\alpha_{i}}: i<n^{*}-1\right)$ of length $j$ we may perform the shrinking of $p\left(\alpha_{n^{*}-1}\right) \uparrow j$ as determined by $p\left(\sigma_{\alpha_{i}}: i<n^{*}-1\right) \uparrow \alpha_{n^{*}-1}$ in $V$. Hence we may obtain $q\left(\alpha_{n^{*}-1}\right)$ such that $p_{n^{*}-1}:=p\left\lceil\alpha_{n^{*}-1} \wedge\left\langle q\left(\alpha_{n^{*}-1}\right)\right\rangle^{\wedge} p\left\lceil\left[\alpha_{n^{*}-1}+1, \omega_{2}\right)\right.\right.$ is $(F, j)$-determined.

Now suppose that for some $r<n^{*}-1$ we have already obtained $(F, j)$-determined $p_{r+1} \leq_{\left\{\alpha_{r+1}, \ldots, \alpha_{n^{*}-1}\right\}}^{*\left[n^{*}, j-1\right]} p$, such that for every $p_{r+1}$-possible $\left(\sigma_{\alpha}: \alpha \in F\right)$ of length $j$ the following holds:

(1) If $i^{*}=\min \left\{j, \min \left\{i: a_{i} \geq d\left(\sigma_{\alpha}: \alpha \in F\right)\right\}\right\}$, then

$$
\begin{aligned}
& p_{r+1}\left(\sigma_{\alpha_{0}}, \ldots, \sigma_{\alpha_{r}}, \sigma_{\alpha_{r+1}} \uparrow i^{*}, \ldots, \sigma_{\alpha_{n^{*}-1}} \uparrow i^{*}\right) \\
& \quad \Vdash \quad \dot{d}=d\left(\sigma_{\alpha}: \alpha \in F\right) \wedge \pi_{\dot{d}}=\pi\left(\sigma_{\alpha}: \alpha \in F\right) " .
\end{aligned}
$$


Note that $i^{*} \geq n^{*}$; hence $p$ and so also $p_{r+1}$ is $\left(F, i^{*}\right)$-determined. Therefore

$$
p_{r+1}\left(\sigma_{\alpha_{0}}, \ldots, \sigma_{\alpha_{r}}, \sigma_{\alpha_{r+1}}\left\lceil i^{*}, \ldots, \sigma_{\alpha_{n^{*}-1}}\left\lceil i^{*}\right)\right.\right.
$$

is well-defined. Fix some $p_{r+1}$-possible $\left(\sigma_{\alpha_{i}}: i \leq r\right)$ of length $j$ and let $\nu=\sigma_{\alpha_{r}}$ । $j-1$. Since $p_{r+1}$ is $(F, j-1)$-determined, there are no more than $N_{1}(j-1)$ many $\left(\nu_{\alpha_{r+1}}, \ldots, \nu_{\alpha_{n^{*}-1}}\right)$, each $\nu_{i}$ being of length $j-1$, such that

$$
\left(\sigma_{\alpha_{0}}, \ldots, \sigma_{\alpha_{r}}, \nu_{\alpha_{r+1}}, \ldots, \nu_{\alpha_{n^{*}-1}}\right)
$$

is $p_{r+1}$-possible. For each $n^{*} \leq k \leq j-1$ we label $\left(\nu_{\alpha_{r+1}} \uparrow k, \ldots, \nu_{\alpha_{n^{*}-1}} \uparrow k\right)$ either with $*$ in the case that

$$
p_{r+1}\left(\sigma_{\alpha_{0}}, \ldots, \sigma_{\alpha_{r}}, \nu_{\alpha_{r+1}}\left\lceil k, \ldots, \nu_{\alpha_{n^{*}-1}}\lceil k) \Vdash “ \dot{d}>a_{k} ",\right.\right.
$$

or else with $(d, \pi)$ such that $d \leq a_{k}$ and

$$
p_{r+1}\left(\sigma_{\alpha_{0}}, \ldots, \sigma_{\alpha_{r}}, \nu_{\alpha_{r+1}}\left\lceil k, \ldots, \nu_{\alpha_{n^{*}-1}}\lceil k) \Vdash " \dot{d}=d \wedge \pi_{\dot{d}}=\pi " .\right.\right.
$$

By (1), every $\left(\nu_{\alpha_{r+1}}\left\lceil k, \ldots, \nu_{\alpha_{n^{*}-1}}\lceil k)\right.\right.$ gets labelled in this way. Moreover, there are no more than $N_{2}(j-1)$ possible labellings of $\left(\nu_{\alpha_{r+1}}\left\lceil k, \ldots, \nu_{\alpha_{n^{*}-1}}\lceil k)\right.\right.$.

Therefore, using the $N_{2}(j-1)^{(j-1) N_{1}(j-1)}$-completeness of \|\|$_{j}$ we may shrink $\operatorname{succ}_{p_{r+1}\left(\alpha_{r}\right)}(\nu)$, by dropping the norm by at most 1 , to some set $S$ such that the label of any fixed $p_{r+1}\left(\sigma_{\alpha_{0}}, \ldots, \sigma_{\alpha_{r-1}}, \sigma\right)$-possible $\left(\nu_{\alpha_{r+1}}, \ldots, \nu_{\alpha_{n^{*}-1}}\right)$ of length $k$, $n^{*} \leq k \leq j-1$, is constant as we vary $\sigma \in S$.

By doing this shrinking for each $\operatorname{succ}_{p_{r+1}\left(\alpha_{r}\right)}(\nu)$, for $\nu \in p_{r+1}\left(\alpha_{r}\right)\lceil j-1$ as determined by $\left(\sigma_{\alpha_{0}}, \ldots, \sigma_{\alpha_{r-1}}\right)$, and for every $p_{r+1}$-possible $\left(\sigma_{\alpha_{0}}, \ldots, \sigma_{\alpha_{r-1}}\right)$ of length $j$, we obtain $p_{r+1}^{j} \leq^{*[j-1]} p_{r+1}$. Note that then for every $p_{r+1}^{j}$-possible $\left(\sigma_{\alpha}: \alpha \in F\right)$ of length $j$ the following holds:

$(1)_{j}$ if $i^{*}$ is minimal such that $a_{i^{*}} \geq d\left(\sigma_{\alpha}: \alpha \in F\right)$, then $i^{*} \leq j-1$ implies that $p_{r+1}^{j}\left(\sigma_{\alpha_{0}}, \ldots, \sigma_{\alpha_{r-1}}, \sigma_{\alpha_{r}}\left\lceil j-1, \sigma_{\alpha_{r+1}}\left\lceil i^{*}, \ldots, \sigma_{\alpha_{n^{*}-1}} \uparrow i^{*}\right)\right.\right.$ decides $\dot{d}$ and $\pi_{\dot{d}}$ (as $d\left(\sigma_{\alpha}: \alpha \in F\right)$ and $\pi\left(\sigma_{\alpha}: \alpha \in F\right)$.

Completely analogously we continue to shrink level $j-2$ of $p_{r+1}^{j}\left(\alpha_{r}\right)$ to obtain $p_{r+1}^{j-1}$, and so on, until we obtain $p_{r+1}^{n^{*}+1}$ such that for every $j \geq i \geq n^{*},(1)_{i}$ holds, where $(1)_{i}$ is $(1)_{j}$ with $j$ replaced by $i$. Then let $p_{r}=p_{r+1}^{n^{*}+1}$. In this way, finally we obtain $p_{0}$, which we call $q$. Then $q$ is as desired.

Corollary 3.7. Let $\Pi=\left(\pi_{d}: d \in \dot{D}\right)$ be a $P_{\omega_{2}}$-name for a predictor for the space $\prod_{n<\omega} g(n)$, and let $p \in P_{\omega_{2}}$. Then there exist $q \leq p$ and $x \in \prod_{n<\omega} g(n)$ in $V$ such that $q \Vdash$ " $\Pi$ does not predict $x$ ".

Proof. Let $(\dot{d}(n): n<\omega)$ increasingly enumerate $\dot{D}$, and let the sequences $\left(F_{n}\right.$ : $n<\omega)$ and $\left(j_{n}: n<\omega\right)$, and $q_{0} \leq p$ be obtained by Corollary 3.5.

By applying Lemma 3.6 repeatedly we shall construct a sequence $\left(q_{0}^{n}: n<\omega\right)$ such that the following hold:

(1) $q_{0}^{0}=q_{0}$,

(2) letting $m^{*}(n)$ be the least $m$ such that $a_{m} \geq \min \left\{d^{n}\left(\sigma_{\alpha}: \alpha \in F_{n+1}\right):\left(\sigma_{\alpha}\right.\right.$ : $\left.\alpha \in F_{n+1}\right)$ is $q_{0}$-possible of length $\left.j_{n+1}\right\}$, we have

$$
q_{0}^{n+1} \leq_{F_{n+1}}^{*\left[m^{*}(n), j_{n+1}-1\right]} q_{0}^{n},
$$


(3) for every $q^{n+1}$-possible $\left(\sigma_{\alpha}: \alpha \in F_{n+1}\right)$ of length $j_{n+1}$, if $i^{*}=\min \left\{j_{n+1}\right.$, $\left.\min \left\{i: a_{i} \geq d^{n}\left(\sigma_{\alpha}: \alpha \in F_{n+1}\right)\right\}\right\}$, then

$$
\begin{aligned}
& q_{0}^{n+1}\left(\sigma_{\alpha}\left\lceil i^{*}: \alpha \in F_{n+1}\right)\right. \\
& \quad \Vdash " \dot{d}\left(a_{j_{n}}\right)=d^{n}\left(\sigma_{\alpha}: \alpha \in F_{n+1}\right) \wedge \pi_{\dot{d}\left(a_{j_{n}}\right)}=\pi^{n}\left(\sigma_{\alpha}: \alpha \in F_{n+1}\right) " .
\end{aligned}
$$

For the construction at step $n$, clearly we have $\Vdash " \dot{d}\left(a_{j_{n}}\right)>a_{j_{n}-1}$ ", and hence $m^{*}(n)>j_{n}-1$. Hence $m^{*}(n) \geq j_{n} \geq n+1=\left|F_{n+1}\right|$, so Lemma 3.6 applies to give $q_{0}^{n+1}$.

Since we have that $\left[m^{*}(n), j_{n+1}-1\right] \cap\left[m^{*}\left(n^{\prime}\right), j_{n^{\prime}+1}-1\right]=\emptyset$ for all $n \neq n^{\prime}$, it follows that the sequence $\left(q_{0}^{n}: n<\omega\right)$ has an infimum, which we call $q$.

Now fix $n$, let $q$-possible $\left(\sigma_{\alpha}: \alpha \in F_{n+1}\right)$ of length $j_{n+1}$ be arbitrary, and let $d=d^{n}\left(\sigma_{\alpha}: \alpha \in F_{n+1}\right)$ and $i^{*}=\min \left\{j_{n+1}, \min \left\{i: a_{i} \geq d\right\}\right\}$. Then by construction there are at most $\left[\prod_{0<i \leq i^{*}}\left(\begin{array}{c}f\left(a_{i}\right) \\ a_{i}\end{array}\right)\right]^{\left|F_{n+1}\right|}$ many functions $\pi: \prod_{i<d} g(i) \rightarrow g(d)$ such that $\pi=\pi^{n}\left(\sigma_{\alpha}: \alpha \in F_{n+1}\right)$ for some $q$-possible $\left(\sigma_{\alpha}: \alpha \in F_{n+1}\right)$ of length $j_{n+1}$. Moreover, $d \geq a_{j_{n}}>n+1$. By Definition 1.5(ii) we have that

$$
\prod_{i<d} g(i) \cdot \prod_{0<i \leq i^{*}}\left(\begin{array}{c}
f\left(a_{i}\right) \\
a_{i}
\end{array}\right)^{n+1}<g(d) .
$$

Therefore we can choose $k(d) \in g(d)$ not in the range of any of the $\pi^{n}\left(\sigma_{\alpha}: \alpha \in\right.$ $\left.F_{n+1}\right)$ as above. By Corollary 3.5(4), every $d<\omega$ is of the form $d^{n}\left(\sigma_{\alpha}: \alpha \in F_{n+1}\right)$ for at most one $n$. Therefore it is now easy to construct $x \in \prod_{n<\omega} g(n)$ in $V$ such that for every $d<\omega$, if $d=d^{n}\left(\sigma_{\alpha}: \alpha \in F_{n+1}\right)$ for some $n$ and $q$-possible $\left(\sigma_{\alpha}: \alpha \in F_{n+1}\right)$ of length $j_{n+1}$, then $x(d)=k(d)$. By construction we conclude that

$$
q \Vdash "(\forall n) \pi_{\dot{d}\left(a_{j_{n}}\right)}\left(x \uparrow \dot{d}\left(a_{j_{n}}\right)\right) \neq x\left(\dot{d}\left(a_{j_{n}}\right)\right) " .
$$

Then clearly, $q \Vdash$ " $\Pi$ does not predict $x "$.

Definition 3.8. Following [Br], we call an uncountable set $X \subseteq \prod_{n<\omega} g(n)$ a Luzin set of evading functions, if every predictor for the space $\prod_{n<\omega} g(n)$ predicts only countably many members of $X$.

It is clear that the existence of a Luzin set for $\prod_{n<\omega} g(n)$ implies that $\mathfrak{e}_{g}=\omega_{1}$. Hence the following theorem is stronger than Theorem 1.9.

Theorem 3.9. If $g$ runs away from $P(f)$, then in the model of Theorem 1.9 there exists a Luzin set of evading functions for the space $\prod_{n<\omega} g(n)$, whereas $\mathfrak{e}_{f}=\omega_{2}$, and hence there exists no Luzin set of evading functions for $\prod_{n<\omega} f(n)$.

Proof. The proof combines the proof of 1.9 with an argument on proper Suslin forcing in [ShSp2, Section 3]. We only give a sketch. Let $V$ be a model of $C H$. Let $\left(\left(p_{\alpha}, \Pi_{\alpha}\right): \alpha<\omega_{1}\right)$ list all pairs $(p, \Pi)$ such that there exists $\alpha<\omega_{1}$ with $p \in P_{\alpha}, \Pi \in V^{P_{\alpha}}$, and $p \Vdash$ " $\Pi$ is a predictor for $\prod_{n<\omega} g(n)$ ". We construct $X=\left\{x_{\alpha}: \alpha<\omega_{1}\right\}$ inductively such that for every $\alpha<\omega_{1}$, for every $\beta<\alpha$ we have that $x_{\alpha} \neq x_{\beta}$ and it is not the case that $p_{\beta} \Vdash$ " $\Pi_{\beta}$ predicts $x_{\alpha}$ ". For this we only use a variation of the proof of Corollary 3.7 .

We claim that $X$ is as desired. Otherwise there exist $p \in P_{\omega_{2}}$ and $\Pi$ a $P_{\omega_{2}}$-name for a predictor for the space $\prod_{n<\omega} g(n)$ such that $p \Vdash$ " $\Pi$ predicts uncountably many $x \in X$ ". We may assume that $p$ and $\Pi$ are hereditarily countable. Let $\operatorname{cl}(p) \subseteq \omega_{2}$ be the set of coordinates used for the construction of $p$, similarly for 
$\operatorname{cl}(\Pi)$. We may assume that $\operatorname{cl}(\Pi) \subseteq \operatorname{cl}(p)$. Let $a^{*}=\operatorname{cl}(p)$. Let $\delta=$ o.t. $\left(a^{*}\right)$. So $\delta<\omega_{1}$. Let $P_{a^{*}}$ be a countable support iteration of $Q(f, g, A)$ with domain $a^{*}$. So $P_{a^{*}}$ is isomorphic to $P_{\delta}$. By arguments similar to those of [ShSp2, Section 3] we may assume that $p \in P_{a^{*}}$ and $\Pi$ is a $P_{a^{*}}$-name. Identifying $P_{a^{*}}$ and $P_{\delta}$, we find that there exists $\alpha^{\prime}<\omega_{1}$ such that $(p, \Pi)=\left(p_{\alpha^{\prime}}, \Pi_{\alpha^{\prime}}\right)$. Then clearly the following claim contradicts the construction of $X$.

Claim. $p \Vdash_{P_{a^{*}}}$ " $\Pi$ predicts uncountably many $x \in X$ ".

Proof. Otherwise there exist $p_{1} \in P_{a^{*}}$ and $\alpha<\omega_{1}$ such that $P_{a^{*}} \models p_{1} \leq p$ and $p_{1} \Vdash_{P_{a^{*}}}$ " $\Pi$ predicts no $x \in\left\{x_{\beta}: \beta>\alpha\right\}$ ". Choose a countable $N \prec H(\chi), \chi$ large enough, such that $p, p_{1}, \Pi, X, \alpha \in N$. Then of course $N \models \alpha$ is countable. By [ShSp2, Lemma 3.11] there exist $q \in P_{a^{*}}$ and a $P_{a^{*} \text {-name }} \overline{\mathbf{r}}^{\prime}=\left(\mathbf{r}_{\nu}^{\prime}: \nu<\omega_{2}\right)$ such that $P_{a^{*}} \models q \leq p_{1}$ and, letting $\overline{\mathbf{r}}_{a^{*}}=\left(\mathbf{r}_{\nu}: \nu \in a^{*}\right)$ be a $P_{a^{*}}$-name for the $P_{a^{*} \text {-generic reals, we have that }}$

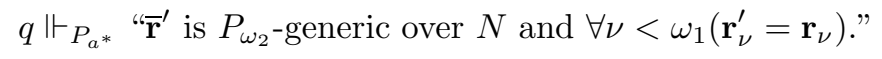

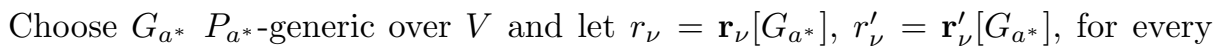
$\nu \in a^{*}, \nu<\omega_{2}$ respectively. Then $r_{\nu}=r_{\nu}^{\prime}$, for all $\nu \in a^{*}$. Hence if $G$ is the $P_{\omega_{2}}{ }^{-}$ generic filter over $N$ determined by $\left(r_{\nu}: \nu<\omega_{2}\right)$, then $p \in G$. By elementarity, $N[G] \models \Pi[G]$ predicts uncountably many $x \in X$. Since $\alpha$ is countable in $N$, this implies that $N[G] \models \Pi[G]$ predicts $x_{\beta}$ for some $\beta>\alpha$. On the other hand, since $p_{1} \in G_{a^{*}}, V\left[G_{a^{*}}\right] \models \Pi\left[G_{a^{*}}\right]$ predicts no $x \in\left\{x_{\gamma}: \gamma>\alpha\right\}$. But $\Pi[G]=\Pi\left[G_{a^{*}}\right]$, since for the evaluation of $\Pi$ only coordinates in $a^{*}$ are used and there $G$ and $G_{a^{*}}$ essentially agree, i.e. determine the same generic reals. Since $N[G] \subseteq V\left[G_{a^{*}}\right]$, we have a contradiction.

Remark 3.10. The crucial [ShSp2, Lemma 3.11] is only proved for Mathias forcing. However it can easily be checked that it carries over to $Q(f, g, A)$.

\section{REFERENCES}

[B] A. Blass, Cardinal characteristics and the product of countably many infinite cyclic groups, Journal of Algebra, vol.169 (1994), pp.512-540. MR 95h:20069

[Br] J. Brendle, Evasion and prediction - the Specker phenomenon and Gross spaces, Forum Mathematicum, vol.7 (1995), pp.513-541. MR 96i:03042

[BrSh] J. Brendle and S. Shelah, Evasion and prediction II, Journal of the London Mathematical Society, vol.53, (1996), pp.19-27. MR 97d:03061

[GSh] M. Goldstern and S. Shelah, Many simple cardinal invariants, Archive for Mathematical Logic, vol.32 (1993), pp.203-221. MR 94c:03064

[L] C. Laflamme, Combinatorial aspects of $F_{\sigma}$ filters with an application to $\mathcal{N}$-sets, Proc. AMS, to appear. CMP 96:16

[RSh1] A. Roslanowski and S. Shelah, Localizations of infinite subsets of $\omega$, Arch. Math. Logic 35 (1996), 315-339. CMP 97:04

[RSh2] A. Roslanowski and S. Shelah, Norms on possibilities I, preprint, pp.1-186, (publication 470 in Shelah's list of publications).

[Sh1] S. Shelah, On cardinal invariants of the continuum, in: Contemporary Mathematics, vol.31 (1984), pp.183-207. MR 86b:03064

[Sh2] S. Shelah, Vive la difference I: Nonisomorphism of ultrapowers of countable models, in: Set theory of the continuum, MSRI publications, vol.26 (1992), pp.357-405. MR 94g:03068

[Sh3] S. Shelah, Proper forcing, Lecture Notes in Math., vol. 940, Springer-Verlag (1982). MR 84h:03002

[ShSp1] S. Shelah and O. Spinas, Gross spaces, Trans. AMS, vol.348 (1996), pp.4257-4277. MR 96m:03031 
[ShSp2] S. Shelah and O. Spinas, The distributivity numbers of $\mathcal{P}(\omega) /$ fin and its square, Trans. AMS, to appear.

[Sp] O. Spinas, Cardinal invariants and quadratic forms, in: Israel Mathematical Conference Proceedings, vol.6 (1993), pp.563-581. MR 94f:03059

Mathematik, ETH-Zentrum, 8092 Zürich, Switzerland

E-mail address: spinas@math.ethz.ch

Current address: Institut für Mathematik, Humboldt-Universität, Unter den Linden 6, 10099 Berlin, Germany 